\title{
ASYMPTOTIC BEHAVIOR FOR THE FILTRATION EQUATION IN DOMAINS WITH NONCOMPACT BOUNDARY
}

\author{
DANIELE ANDREUCCI AND ANATOLI F. TEDEEV
}

\begin{abstract}
We consider the initial value boundary problem with zero Neumann data for an equation modelled after the porous media equation, with variable coefficients. The spatial domain is unbounded and shaped like a (general) paraboloid, and the solution $u$ is integrable in space and non-negative. We show that the asymptotic profile for large times of $u$ is one-dimensional and given by an explicit function, which can be regarded as the fundamental solution of a one-dimensional differential equation with weights.

In the case when the domain is a cone or the whole space (Cauchy problem) we obtain a genuine multi-dimensional profile given by the well known Barenblatt solution.

This is an Accepted Manuscript version of the following article, accepted for publication in Comm. Partial Differential Equations 42 (2017), no. 3, 347-365. It is deposited under the terms of the Creative Commons Attribution-NonCommercial License (http://creativecommons.org/licenses/by-nc/4.0/), which permits non-commercial re-use, distribution, and reproduction in any medium, provided the original work is properly cited. Available on-
\end{abstract} line: http://www.tandfonline.com/10.1080/03605302.2017.1278770

\section{INTRODUCTION}

Consider in $S_{T}=\Omega \times(0, T)$ the following Neumann problem for the filtration equation

$$
\begin{aligned}
\frac{\partial u}{\partial t}-\frac{\partial}{\partial x_{j}}\left(a_{i j}(x, t) \frac{\partial u^{m}}{\partial x_{i}}\right) & =0, & & \text { in } S_{T}=\Omega \times(0, T), \\
a_{i j}(x, t) \frac{\partial u^{m}}{\partial x_{i}} \nu_{j} & =0, & & \text { on } \partial \Omega \times(0, T), \\
u(x, 0) & =u_{0}(x), & & x \in \Omega .
\end{aligned}
$$

AMS Classification: 35K55, 35K65, 35B40.

The first author is member of the Gruppo Nazionale per la Fisica Matematica (GNFM) of the Istituto Nazionale di Alta Matematica (INdAM). . 
Notice that we understand throughout summation with respect to repeated indexes. Here $\Omega \subset \boldsymbol{R}^{N}$ is a domain with sufficiently smooth non compact boundary, $N \geq 2, m>1, \nu=\left(\nu_{1}, \ldots, \nu_{N}\right)$ is the unit outer normal to $\partial \Omega, u_{0} \in L^{1}\left(\boldsymbol{R}^{N}\right)$ is a non-negative function, and the coefficients $a_{i j} \in L^{\infty}\left(S_{T}\right), i, j=1, \ldots N,(x, t) \in S_{T}$ are such that for a constant $c_{0} \geq 1$ and for all $\xi=\left(\xi_{1}, \ldots, \xi_{N}\right) \in \boldsymbol{R}^{N}$, a.e. $(x, t) \in S_{\infty}$ we have

$$
c_{0}^{-1}|\xi|^{2} \leq a_{i j}(x, t) \xi_{i} \xi_{j} \leq c_{0}|\xi|^{2} .
$$

The main goal of this paper is to get the asymptotic profile as $t \rightarrow \infty$ of solutions to (1.1)-(1.3) in $S=S_{\infty}$ under additional assumptions on the coefficients and on the geometry of the domain $\Omega$ (see (1.8)).

Since (1.1) is degenerate on $\{u=0\}$ we must understand the concept of solution in a weak sense. We denote by $B_{R}(x)$ the ball of radius $R$ centered at $x \in \boldsymbol{R}^{N}$.

Definition 1.1. We say that $u \geq 0$ is a weak solution of (1.1)-(1.3) in $S_{T}$ if

$$
\begin{gathered}
u \in C\left((0, T) ; L^{1}(\Omega)\right) \cap L_{\mathrm{loc}}^{\infty}\left((0, T) ; L^{\infty}(\Omega)\right), \\
\left|\nabla u^{m}\right| \in L^{1}\left(S_{T}\right) \cap L_{\mathrm{loc}}^{2}\left(0, T ; L^{2}\left(\Omega \cap B_{R}(0)\right)\right), \quad \text { for all } R>0,
\end{gathered}
$$

and for any test function $\varphi \in C^{\infty}\left(\overline{S_{T}}\right)$ with $\varphi(x, T)=0$ the following identity holds:

$$
\iint_{S_{T}}\left(-u \varphi_{t}+a_{i j}(x, t)\left(u^{m}\right)_{x_{i}} \varphi_{x_{j}}\right) \mathrm{d} x \mathrm{~d} t=\int_{\Omega} u(x, 0) \varphi(x, 0) \mathrm{d} x .
$$

It is well known (see [5]) that a weak solution in the sense of Definition 1.1 is in fact locally Hölder continuous in $S_{T}$.

If $a_{i j}(x, t)=\delta_{i j}$ where $\delta_{i j}$ denotes the Kronecker symbol, then (1.1) is the porous media equation (PME). It is well known (see [13], [18]) that if $\Omega=\boldsymbol{R}^{N}$ and $u_{0} \in L_{1}\left(\boldsymbol{R}^{N}\right)$ then as $t \rightarrow \infty$ we have

$$
t^{\frac{N}{\beta}}|u(x, t)-E(x, t)| \rightarrow 0,
$$

uniformly in $x \in \boldsymbol{R}^{N}$, where $\beta=N(m-1)+2$ is the so called Barenblatt exponent and $E(x, t)$ is the fundamental solution of the PME with the same total mass as $u_{0}(x)$. More explicitly

$$
E(x, t)=t^{-\frac{N}{\beta}}\left(C-c(m, N)\left(\frac{|x|}{t^{1 / \beta}}\right)^{2}\right)_{+}^{\frac{1}{m-1}} .
$$


Here $c(m, N)=(2 \beta)^{-1}(m-1)$ and $C$ is chosen so that $\left\|u_{0}\right\|_{1}=\|E(t)\|_{1}$ for $t>0$. Note that in the aforementioned papers the uniqueness of the fundamental solution plays an important role. Such a result was proven first in [15] and in [13] with alternative approaches. More recently the approach of [13] was simplified in [16]. In the case of bounded domains we quote the recent paper [7].

1.1. Geometry of the domain. Let us turn now to our problem. We define namely the paraboloid-like domains

$$
\Omega(\alpha)=\left\{x \in \boldsymbol{R}^{N}\left|x_{N}>0,\right| x^{\prime} \mid<x_{N}^{\alpha}\right\},
$$

where $0 \leq \alpha \leq 1$ and $x^{\prime}=\left(x_{1}, \ldots, x_{N-1}\right)$. Actually the two extreme cases $\alpha=0$ and $\alpha=1$ hardly deserve the definition of paraboloid-like domain, corresponding respectively to the case of the cylinder and of the cone.

Essentially, we prove that when $\alpha<1$ the asymptotic profile of the solution to (1.1)-(1.3) is one-dimensional. When $\alpha=1$ instead the profile is genuinely $\mathrm{N}$-dimensional; in this instance our results cover also the case of the Cauchy problem. Indeed to the best of our knowledge, for equations containing variable coefficients $a_{i j}$, even the latter case was not treated in the literature, so we believe our approach is of interest in view both of the geometry of the domain and of the structure of the equation it allows.

For this choice of $\Omega$ in (1.1)-(1.3) we know from [3] that a solution $u$ satisfies

$$
\|u(t)\|_{\infty} \leq \gamma \max \left\{\frac{\left\|u_{0}\right\|_{1}^{2 / \beta}}{t^{N / \beta}}, \frac{\left\|u_{0}\right\|_{1}^{2 / b(\alpha)}}{t^{n(\alpha) / b(\alpha)}}\right\}, \quad \text { for any } t>0 .
$$

Here and below we denote by $\gamma$ a generic positive constant depending only on the parameters $N, m, \alpha$, and use the notation for $-1 /(N-1)<$ $s \leq 1$

$$
\begin{gathered}
n(s)=s(N-1)+1, \quad b(s)=n(s)(m-1)+2, \\
\lambda(s)=N b(s) \beta^{-1}-n(s)>0, \quad \text { if } s<1,
\end{gathered}
$$

and let $\beta=b(1)=N(m-1)+2$ be the usual Barenblatt number. Notice that $n(\alpha)$ is nothing but the dimension at infinity of $\Omega(\alpha)$, while $n(1)=N$ is the topological dimension of $\Omega(\alpha)$. This remark is made precise by the equality

$$
\left|\Omega_{R}\right|=\frac{\omega_{N-1}}{n(\alpha)} R^{n(\alpha)}, \quad R>0, \quad \Omega_{R}:=\Omega(\alpha) \cap\left\{x_{N}<R\right\},
$$


where $\omega_{N-1}$ is the $(N-1)$-dimensional measure of the unit ball in $\boldsymbol{R}^{N-1}$, and by the observation that the asymptotics for large $R$ of $\left|\Omega(\alpha) \cap B_{R}(0)\right|$ are given by the same power function as in (1.10).

Remark 1.2. Some general remarks on the interplay between the geometry of the domain and the behavior of solutions to partial differential equations like (1.1) are perhaps in order. The classical approach to the existence theory of solutions to problem (1.1)-(1.3) (see for example [19]) relies upon suitable embedding results yielding compactness of a sequence of compactly supported approximating solutions. In turn, embedding results rely on isoperimetric inequalities, which leads us to consider the classes of domains treated in $[8,9,10],[3,4]$. For domains in these classes the $L^{\infty}$ estimates which are the main tool in our approach were proven in $[2,3,4]$, for a wide class of Neumann problems for doubly degenerate parabolic equations including (1.1). However in this paper we deal only with model domains as in (1.8), which dispenses us from giving here the general definitions of the admissible classes. This restriction allows us in fact to apply rescaling arguments without cumbersome complications.

Next we introduce the following fundamental solution which appears in our main result as the one-dimensional asymptotic profile; let

$$
E_{\alpha}(y, t)=t^{-\frac{n(\alpha)}{b(\alpha)}}\left(C-\frac{m-1}{2 m b(\alpha)}\left(\frac{y}{t^{\frac{1}{b(\alpha)}}}\right)^{2}\right)_{+}^{\frac{1}{m-1}}, \quad t>0, y>0 .
$$

Then, for a suitable choice of $C>0, E_{\alpha}$ solves the problem

$$
\begin{aligned}
y^{(N-1) \alpha} \frac{\partial V}{\partial t}-\frac{\partial}{\partial y}\left(y^{(N-1) \alpha} \frac{\partial V^{m}}{\partial y}\right) & =0, & & t>0, y>0, \\
y^{(N-1) \alpha} \frac{\partial V^{m}}{\partial y} & =0, & & t>0, y=0, \\
y^{(N-1) \alpha} V(y, 0) & =M \delta(y), & & y>0,
\end{aligned}
$$

where $\delta$ denotes Dirac's mass, and we let

$$
M=\omega_{N-1}^{-1}\left\|u_{0}\right\|_{1} .
$$

It is straightforward to check that $E_{\alpha}$ solves (1.12)-(1.14) in the following sense. 
Definition 1.3. A function $V:(0,+\infty) \times(0,+\infty) \rightarrow[0,+\infty)$ is a weak solution to $(1.12)-(1.14)$, if for $\mathrm{d} q=y^{(N-1) \alpha} \mathrm{d} y$,

$$
\begin{gathered}
V,\left(V^{m}\right)_{y} \in L^{1}((0, R) \times(0, T) ; \mathrm{d} q) ; \\
\left(V^{m}\right)_{y} \in L^{2}((0, R) \times(\tau, T) ; \mathrm{d} q),
\end{gathered}
$$

for all $R>0, T>\tau>0$, In addition we ask that

$$
\int_{0}^{+\infty} \int_{0}^{+\infty} y^{(N-1) \alpha}\left(-V \varphi_{t}+\left(V^{m}\right)_{y} \varphi_{y}\right) \mathrm{d} y \mathrm{~d} t=M \varphi(0,0),
$$

for all $\varphi \in C^{1}([0,+\infty) \times[0,+\infty]), \varphi(y, t)=0$ when $y \geq R$ or $t \geq T$, for suitable $R, T>0$.

In fact $V=E_{\alpha}$ is immediately seen to satisfy also, for a suitable $c_{1}>0$,

$$
\begin{aligned}
V(y, t) & \leq c_{1} t^{-\frac{n(\alpha)}{b(\alpha)}}, & & y>0, t>0: \\
V(y, t) & =0, & & y \geq c_{1} t^{\frac{1}{b(\alpha)}} ; \\
\|V(t)\|_{L^{1}(0,+\infty ; \mathrm{d} q)} & =M, & & t>0 .
\end{aligned}
$$

This fact will be relevant in the proof of the following uniqueness result, which is essential to us.

Theorem 1.4. Any solution $V$ to (1.12)-(1.14) in the sense of Definition 1.3 coincides with $E_{\alpha}$, provided $V$ also fulfills requirements (1.18)(1.20).

1.2. Main results. Further we need some assumptions on the asymptotic behavior of the coefficients $a_{i N}, i=1, \ldots, N$. Let $\delta_{i N}, i=1, \ldots$, $N$ be the standard Kronecker symbol. Then we assume for $i=1, \ldots$, $N$ that

$$
\lim _{\rho \rightarrow \infty} \rho^{-(n(\alpha)+b(\alpha))} \int_{0}^{\rho^{b(\alpha)}} \int_{\Omega_{\rho}}\left|a_{i N}(y, t)-\delta_{i N}\right|^{2} \mathrm{~d} y \mathrm{~d} t=0 .
$$

We remark that the quantity appearing in (1.21) is essentially an integral average.

Before stating our main results we introduce the following notation: the set

$$
P_{R}=\left\{(x, t)|x \in \Omega(\alpha)| x_{N}<R t^{\frac{1}{b(\alpha)}}\right\},
$$

for any given $R>0$ is called interior domain, while $S_{\infty} \backslash \overline{P_{R}}$ is called outer domain. As far as we know, no general uniqueness result is 
available for our problem; thus in the Theorems below we refer to any solution obtained as outlined in Remark 1.2.

Our first result is

Theorem 1.5. Let $u$ be a solution of (1.1)-(1.3) in $S_{\infty}=\Omega(\alpha) \times$ $(0, \infty)$ with $0 \leq \alpha<1$ in the sense above, and let $E_{\alpha}$ be as in (1.11).

Assume that $\left(a_{i N}\right)$ fulfills condition (1.21). Then $u$ approaches $E_{\alpha}$ as $t \rightarrow+\infty$ in the following sense, for all $p \in[1,+\infty)$.

INTERIOR DOMAIN: For all $R>0, T>\tau>0$,

$$
t^{\frac{n(\alpha)}{b(\alpha)} p} f_{t \tau}^{t T} f_{\Omega_{t^{1 / b(\alpha)}}}\left|u(y, s)-E_{\alpha}\left(y_{N}, s\right)\right|^{p} \mathrm{~d} y \mathrm{~d} s \rightarrow 0 .
$$

If in addition $a_{i j}=a_{i j}(x)$,

$$
t^{\frac{n(\alpha)}{b(\alpha)} p} f_{\Omega_{t^{1 / b(\alpha)} R}}\left|u(y, t)-E_{\alpha}\left(y_{N}, t\right)\right|^{p} \mathrm{~d} y \rightarrow 0 .
$$

Outer DOMAIN: For a suitable $\Gamma>0$ :

$$
t^{\frac{n(\alpha)}{b(\alpha)}}\left\|u(t)-E_{\alpha}(t)\right\|_{\infty,\left\{x_{N}>\Gamma t^{1 / b(\alpha)}\right\}} \rightarrow 0 .
$$

Next we consider the case of cones, i.e., $\alpha=1$; in this case the asymptotic profile is $N$-dimensional, in the following sense.

Theorem 1.6. Let $u$ be a solution of (1.1)-(1.3) in $S_{\infty}=\Omega(1) \times$ $(0, \infty)$ in the sense above, and let $E$ be as in $(1.7)$, with $C$ such that $\|E(t)\|_{1, \Omega(1)}=\left\|u_{0}\right\|_{1, \Omega(1)}$.

Assume that $\left(a_{i j}\right)$ fulfills the condition

$$
\lim _{\rho \rightarrow \infty} \rho^{-(N+\beta)} \int_{0}^{\rho^{\beta}} \int_{\Omega_{\rho}}\left|a_{i j}(y, t)-\delta_{i j}\right|^{2} \mathrm{~d} y \mathrm{~d} t=0, \quad 1 \leq i, j \leq N .
$$

Then we have

$$
t^{\frac{N}{\beta}}\|u(t)-E(t)\|_{\infty} \rightarrow 0, \quad t \rightarrow \infty .
$$

The same result holds true if $\Omega(1)$ is formally replaced with $\boldsymbol{R}^{N}$ above, that is in the case of the Cauchy problem.

In Section 2 we present the proof of Theorem 1.5 and the few changes needed to prove Theorem 1.6. The uniqueness result Theorem 1.4 is proved in Section 3; finally Sections 5 and 4 are devoted to some necessary technical results. 


\section{Proof of Theorem 1.5 .}

In the first steps of the proof we use the rescaling arguments introduced by Kamin in an essential way (see [11, 17, 12, 13, 19, 1, 6] and references therein). In the following we denote $\Omega=\Omega(\alpha)$ for the sake of simplicity.

Let $u_{k}(x, t)=k^{n(\alpha)} u\left(k^{\alpha} x^{\prime}, k x_{N}, k^{b(\alpha)} t\right), k \geq 1$. Then $u_{k}(x, t)$ is a weak solution to the equation

$$
\frac{\partial u_{k}}{\partial t}=\frac{\partial}{\partial x_{j}}\left(A_{i j}^{k}(x, t)\left(u_{k}^{m}\right)_{x_{i}}\right)
$$

where for $i, j=1, \ldots N-1$,

$$
\begin{aligned}
A_{i j}^{k}=k^{2(1-\alpha)} a_{i j}^{k}, \quad & A_{N j}^{k}=k^{1-\alpha} a_{N j}^{k}, \quad A_{j N}^{k}=k^{1-\alpha} a_{j N}^{k}, \quad A_{N N}^{k}=a_{N N}^{k}, \\
& a_{i j}^{k}(x, t)=a_{i j}\left(k^{\alpha} x^{\prime}, k x_{N}, k^{b(\alpha)} t\right) .
\end{aligned}
$$

In addition, also in a standard weak sense,

$$
A_{i j}^{k}(x, t) \frac{\partial u_{k}^{m}}{\partial x_{i}} \nu_{j}=0, \quad \text { on } \partial \Omega \times(0, T),
$$

and

$$
u_{k}(x, 0)=u_{k 0}(x)=k^{n(\alpha)} u_{0}\left(k^{\alpha} x^{\prime}, k x_{N}\right), \quad x \in \Omega .
$$

Notice that for $t>0$

$$
\int_{\Omega} u_{k}(x, t) \mathrm{d} x=\int_{\Omega} u(x, t) \mathrm{d} x=\int_{\Omega} u_{0}(x) \mathrm{d} x .
$$

As a direct consequence of the definition of $u_{k}$ and of estimate (1.9) we have for $k \geq 1$ the estimates for $u_{k}$

$$
\begin{aligned}
\left\|u_{k}(t)\right\|_{\infty} \leq \gamma^{*} & \max \left\{\frac{\left\|u_{0}\right\|_{1}^{\frac{2}{\beta}}}{t^{\frac{N}{\beta}} k^{\lambda(\alpha)}}, \frac{\left\|u_{0}\right\|_{1}^{\frac{2}{b(\alpha)}}}{t^{\frac{n(\alpha)}{b(\alpha)}}}\right\} \\
\leq & \gamma^{*} \max \left\{\frac{\left\|u_{0}\right\|_{1}^{\frac{2}{\beta}}}{t^{\frac{N}{\beta}}}, \frac{\left\|u_{0}\right\|_{1}^{\frac{2}{b(\alpha)}}}{t^{\frac{n(\alpha)}{b(\alpha)}}}\right\}, \text { for all } t>0 .
\end{aligned}
$$

Next we need the following auxiliary result, where we use the notation

$$
\begin{gathered}
S_{T, R}=\Omega_{R} \times(0, T), \quad S_{T, R, \tau}=\Omega_{R} \times(\tau, T), \\
\nabla_{x^{\prime}}=\left(\frac{\partial}{\partial x_{1}}, \ldots, \frac{\partial}{\partial x_{N-1}}\right) .
\end{gathered}
$$

We also understand in the following that indexes denoted by $i^{\prime}$ range over $1, \ldots, N-1$. 
Proposition 2.1. Under the conditions of Theorem 1.5, we have for all $k \geq 1, T>\tau>0, R>0$

$$
\int_{\tau}^{T} \int_{\Omega_{R}}\left|\left(u_{k}^{m}\right)_{x_{N}}\right|^{2} \mathrm{~d} x \mathrm{~d} t+k^{2(1-\alpha)} \int_{\tau}^{T} \int_{\Omega_{R}}\left|\nabla_{x^{\prime}} u_{k}^{m}\right|^{2} \mathrm{~d} x \mathrm{~d} t \leq \gamma,
$$

where the constant on the right-hand side depends on $c_{0},\left\|u_{0}\right\|_{1}, \tau, T$, $R$ but not on $k$.

Proof. Multiply both sides of the equation (2.1) by $u_{k}^{m} \zeta^{2}\left(x_{N}\right)(t-\tau / 2)$ where $\zeta\left(x_{N}\right)$ is a smooth cutoff function in $(0,2 R)$ such that $\zeta=1$ in $[0, R]$ and $\left|\zeta_{x_{N}}\right| \leq \gamma / R$. Then integrating by parts over $S_{T, R}$ we obtain

$$
\begin{aligned}
\int_{\Omega_{2 R}} & \frac{u_{k}(x, T)^{m+1}}{m+1}\left(T-\frac{\tau}{2}\right) \zeta^{2} \mathrm{~d} x \\
& +\int_{\frac{\tau}{2}} \int_{\Omega_{2 R}} A_{i j}^{k}\left(u_{k}^{m}\right)_{x_{i}}\left(u_{k}^{m}\right)_{x_{j}}\left(t-\frac{\tau}{2}\right) \zeta^{2} \mathrm{~d} x \mathrm{~d} t \\
= & \int_{\frac{\tau}{2}}^{T} \int_{\Omega_{2 R}} \frac{u_{k}^{m+1}}{m+1} \zeta^{2} \mathrm{~d} x \mathrm{~d} t \\
& +2 \int_{\frac{\tau}{2}}^{T} \int_{\Omega_{2 R}} A_{i N}^{k}\left(u_{k}^{m}\right)_{x_{i}} u_{k}^{m}\left(t-\frac{\tau}{2}\right) \zeta \zeta_{x_{N}} \mathrm{~d} x \mathrm{~d} t .
\end{aligned}
$$

On applying the Cauchy inequality and (1.4) to this equality we obtain

$$
\begin{aligned}
c_{0} \frac{\tau}{2} \int_{\tau}^{T} \int_{\Omega_{R}}\left|\left(u_{k}^{m}\right)_{x_{N}}\right|^{2} \mathrm{~d} x \mathrm{~d} t+c_{0} \frac{\tau}{2} k^{2(1-\alpha)} \int_{\tau}^{T} \int_{\Omega_{R}}\left|\nabla_{x^{\prime}} u_{k}^{m}\right|^{2} \mathrm{~d} x \mathrm{~d} t \leq \\
\quad \gamma \int_{\frac{\tau}{2}}^{T} \int_{\Omega_{2 R}} u_{k}^{m+1} \zeta^{2} \mathrm{~d} x \mathrm{~d} t+\frac{\gamma}{R^{2}} \int_{\frac{\tau}{2}}^{T} \int_{\Omega_{2 R}} u_{k}^{2 m}\left(t-\frac{\tau}{2}\right) \mathrm{d} x \mathrm{~d} t .
\end{aligned}
$$

The right-hand side of (2.7) can be estimated by (2.5), whence the sought after estimate follows.

Let $\varphi\left(x_{N}, t\right)$ be a smooth function in $[0,+\infty) \times[0,+\infty)$ such that

$$
\varphi\left(x_{N}, t\right)=0, \quad x_{N} \geq R ; \quad \varphi\left(x_{N}, t\right)=0, \quad t \geq T .
$$


Then from the weak formulation of problem (2.1)-(2.3) for $u_{k}$ we obtain

$$
\begin{aligned}
& \iint_{S_{T, R}}\left(-u_{k} \varphi_{t}+a_{N N}^{k}\left(u_{k}^{m}\right)_{x_{N}} \varphi_{x_{N}}\right) \mathrm{d} x \mathrm{~d} t= \\
& \quad-k^{1-\alpha} \iint_{S_{T, R}} a_{i^{\prime} N}^{k}\left(u_{k}^{m}\right)_{x_{i^{\prime}}} \varphi_{x_{N}} \mathrm{~d} x \mathrm{~d} t+\int_{\Omega_{R}} u_{k}(x, 0) \varphi(x, 0) \mathrm{d} x .
\end{aligned}
$$

Our purpose is to take the limit $k \rightarrow \infty$ in (2.8). First we remark that by making use of (2.5) one can prove (following for example [4] Proposition 3.2 that for any $\tau>0$

$$
\int_{0}^{\tau} \int_{\Omega_{R}}\left(u_{k}\left|\varphi_{t}\right|+\left|A_{i N}^{k}(x, t)\left(u_{k}^{m}\right)_{x_{i}} \varphi_{x_{N}}\right|^{p}\right) \mathrm{d} x \mathrm{~d} t \leq \gamma\left(\varphi, u_{0}\right) \tau^{\omega},
$$

where $\gamma$ and $\omega>0$ also depend on $p \in[1,(N m+2) /(N m+1))$, but not on $k$.

Next we have for $0<\tau<T$

$$
\iint_{S_{T, R, \tau}} a_{N N}^{k}\left(u_{k}^{m}\right)_{x_{N}} \varphi_{x_{N}} \mathrm{~d} x \mathrm{~d} t=I+\iint_{S_{T, R, \tau}}\left(u_{k}^{m}\right)_{x_{N}} \varphi_{x_{N}} \mathrm{~d} x \mathrm{~d} t,
$$

where

$$
\begin{aligned}
|I| & =\left|\iint_{S_{T, R, \tau}}\left(a_{N N}^{k}-1\right)\left(u_{k}^{m}\right)_{x_{N}} \varphi_{x_{N}} \mathrm{~d} x \mathrm{~d} t\right| \\
& \leq\left(\iint_{S_{T, R, \tau}}\left(a_{N N}^{k}-1\right)^{2} \mathrm{~d} x \mathrm{~d} t\right)^{\frac{1}{2}}\left(\iint_{S_{T, R, \tau}}\left(u_{k}^{m}\right)_{x_{N}}^{2} \varphi_{x_{N}}^{2} \mathrm{~d} x \mathrm{~d} t\right)^{\frac{1}{2}} \\
& =: J^{\frac{1}{2}} D^{\frac{1}{2}}
\end{aligned}
$$

Let $y^{\prime}=k^{\alpha} x^{\prime}, y_{N}=k x_{N}, s=t k^{b(\alpha)}$. Then

$$
J=k^{-n(\alpha)-b(\alpha)} \int_{\tau k^{b(\alpha)}}^{T k^{b(\alpha)}} \int_{0}^{R k} \int_{\left|y^{\prime}\right| \leq y_{N}^{\alpha}}\left(a_{N N}(y, s)-1\right)^{2} \mathrm{~d} y^{\prime} \mathrm{d} y_{N} \mathrm{~d} s,
$$

so that $J \rightarrow 0$ as $k \rightarrow \infty$ when we take into account our assumption (1.21). Moreover $D$ is bounded uniformly on $k \geq 1$ due to Proposition 2.1. Therefore $I \rightarrow 0$ as $k \rightarrow \infty$. A similar argument, still invoking 
(1.21), proves that as $k \rightarrow \infty$

$$
\begin{aligned}
& \left|k^{1-\alpha} \iint_{S_{T, R, \tau}} a_{i^{\prime} N}^{k}\left(u_{k}^{m}\right)_{x_{i^{\prime}}} \varphi_{x_{N}} \mathrm{~d} x \mathrm{~d} t\right| \leq\left(\iint_{S_{T, R, \tau}}\left(a_{i^{\prime} N}^{k}\right)^{2} \mathrm{~d} x \mathrm{~d} t\right)^{\frac{1}{2}} \\
& \quad \times\left(k^{2(1-\alpha)} \iint_{S_{T, R, \tau}}\left|\nabla_{x^{\prime}} u_{k}^{m}\right|^{2} \varphi_{x_{N}}^{2} \mathrm{~d} x \mathrm{~d} t\right)^{\frac{1}{2}} \rightarrow 0 .
\end{aligned}
$$

In order to prove compactness for $u_{k}^{m}$ in an integral sense, when we take into account the estimate (2.6), we only need obtain a suitable regularity in time. To this end we obtain the following result from combining the bounds obtained for $u_{k}$ in (2.5) and in (2.6), which are uniform with respect to $k \geq 1$, with classical arguments in the theory of parabolic equations.

Lemma 2.2. For every set $S_{T, R, \tau}$ there exists a constant $\gamma$ depending on $R>0, T>\tau>0$, but not on $k$, such that for all $0<h<1$ and $k \geq 1$

$$
\iint_{S_{T, R, \tau}}\left|u_{k}(x, t+h)-u_{k}(x, t)\right|^{m+1} \mathrm{~d} x \mathrm{~d} t \leq \gamma \sqrt{h} .
$$

If in addition we assume $a_{i j}=a_{i j}(x)$, then for $t \in[\tau, T]$

$$
\int_{\Omega_{R}}\left|u_{k}(x, t+h)-u_{k}(x, t)\right| \mathrm{d} x \leq \gamma h^{\frac{1}{2(m+3)}} .
$$

The proof of this Lemma, involving a careful check of the effects of the strong anisotropy in (2.1), is presented in Section 4.

The estimates (2.6) and (2.14) together with the sup bound (2.5) imply compactness of the sequence $u_{k}$ in $L_{\text {loc }}^{2}\left(0, T ; L^{2}\left(\Omega_{R}\right)\right)$. Possibly by extracting a subsequence we then have $u_{k} \rightarrow u_{\infty}$ as $k \rightarrow \infty$ in $L_{\text {loc }}^{2}\left(0, T ; L^{2}\left(\Omega_{R}\right)\right)$, and a.e. in $S_{\infty}$. Moreover we may assume by invoking (2.6) that

$$
\nabla u_{k}^{m} \rightarrow \nabla u_{\infty}^{m}, \quad \text { weakly in } L_{\mathrm{loc}}^{2}\left(0, T ; L^{2}\left(\Omega_{R}\right)\right) ; \quad \nabla_{x^{\prime}} u_{\infty}^{m}=0,
$$

so that $u_{\infty}$ is independent of the $x^{\prime}$ variables. Finally, it follows easily from (2.5) that

$$
\left\|u_{\infty}(t)\right\|_{\infty} \leq \gamma^{*} \frac{\left\|u_{0}\right\|_{1}^{\frac{2}{b(\alpha)}}}{t^{\frac{n(\alpha)}{b(\alpha)}}}, \quad \text { for all } t>0 .
$$


Thus, by taking into account (2.10)-(2.12) we get for $k \rightarrow \infty$

$$
\begin{aligned}
\iint_{S_{T, R, \tau}}\left(-u_{k} \varphi_{t}+a_{N N}^{k}(x, t)\left(u_{k}^{m}\right)_{x_{N}} \varphi_{x_{N}}\right) \mathrm{d} x \mathrm{~d} t \\
\rightarrow \iint_{S_{T, R, \tau}}\left(-u_{\infty} \varphi_{t}+\left(u_{\infty}^{m}\right)_{x_{N}} \varphi_{x_{N}}\right) \mathrm{d} x \mathrm{~d} t
\end{aligned}
$$

Therefore as $k \rightarrow \infty$ we infer from (2.8) and from (2.13), (2.17), (2.9)

$$
\iint_{S_{T, R}}\left(-u_{\infty} \varphi_{t}+\left(u_{\infty}^{m}\right)_{x_{N}} \varphi_{x_{N}}\right) \mathrm{d} x \mathrm{~d} t=\lim _{k \rightarrow \infty} \int_{\Omega_{R}} u_{k}(x, 0) \varphi\left(x_{N}, 0\right) \mathrm{d} x .
$$

Let us identify the limit in the right-hand side of (2.18). We have

$$
\begin{aligned}
\int_{\Omega_{R}} & u_{k}(x, 0) \varphi\left(x_{N}, 0\right) \mathrm{d} x \\
= & \int_{\Omega_{R}} u_{k}(x, 0)\left(\varphi\left(x_{N}, 0\right)-\varphi(0,0)\right) \mathrm{d} x+\varphi(0,0) \int_{\Omega_{R}} u_{k}(x, 0) \mathrm{d} x \\
= & \int_{\Omega_{k R}} u_{0}\left(y^{\prime}, y_{N}\right)\left(\varphi\left(y_{N} / k, 0\right)-\varphi(0,0)\right) \mathrm{d} y+\varphi(0,0) \int_{\Omega_{k R}} u_{0}(y) \mathrm{d} y .
\end{aligned}
$$

Letting $k \rightarrow \infty$ and using the global integrability of $u_{0}$, we obtain

$$
\lim _{k \rightarrow \infty} \int_{\Omega_{R}} u_{k}(x, 0) \varphi\left(x_{N}, 0\right) \mathrm{d} x=\varphi(0,0) \int_{\Omega} u_{0}(x) \mathrm{d} x .
$$

Thus from (2.18), and recalling that the support of $\varphi$ is contained in $S_{T, R}$ we have

$$
\iint_{S_{T, R}}\left(-u_{\infty} \varphi_{t}+\left(u_{\infty}^{m}\right)_{x_{N}} \varphi_{x_{N}}\right) \mathrm{d} x \mathrm{~d} t=\varphi(0,0)\left\|u_{0}\right\|_{1}=\omega_{N-1} \varphi(0,0) M .
$$

Recalling that the limit function is independent of $x^{\prime}$, we infer

$$
\int_{0}^{T} \int_{0}^{\infty} x_{N}^{\alpha(N-1)}\left(-u_{\infty} \varphi_{t}+\left(u_{\infty}^{m}\right)_{x_{N}} \varphi_{x_{N}}\right) \mathrm{d} x_{N} \mathrm{~d} t=M \varphi(0,0) .
$$

Now it follows from (2.19) and from Theorem 1.4, see Corollary 3.1 in Section 3 for the details, that $u_{\infty}=E_{\alpha}$. Therefore, the whole sequence $u_{k}$ tends to $E_{\alpha}$ in $L_{\text {loc }}^{2}\left(0, T ; L^{2}\left(\Omega_{R}\right)\right)$ and actually in $L_{\text {loc }}^{p}\left(0, T ; L^{p}\left(\Omega_{R}\right)\right)$ for all $R$ and $p \geq 1$, owing to the sup bound (2.5). 
2.1. The interior domain. Next fix $R>0, T>\tau>0, p \geq 1$ and compute, by taking advantage of the self-similar form of $E_{\alpha}$, and by changing variables,

$$
\begin{aligned}
I_{k} & =f_{\tau}^{T} f_{\Omega_{R}}\left|u_{k}\left(x^{\prime}, x_{N}, z\right)-E_{\alpha}\left(x_{N}, z\right)\right|^{p} \mathrm{~d} x \mathrm{~d} z \\
& =f_{\tau}^{T} f_{\Omega_{R}}\left|k^{n(\alpha)} u\left(k^{\alpha} x^{\prime}, k x_{N}, k^{b(\alpha)} z\right)-k^{n(\alpha)} E_{\alpha}\left(k x_{N}, k^{b(\alpha)} z\right)\right|^{p} \mathrm{~d} x \mathrm{~d} z \\
& =k^{n(\alpha) p} f_{k^{b(\alpha)} \tau}^{f_{\Omega_{k R}}^{b(\alpha)} T} f u(y, s)-\left.E_{\alpha}\left(y_{N}, s\right)\right|^{p} \mathrm{~d} y \mathrm{~d} s .
\end{aligned}
$$

Now for any given $t \geq 1$ we select $k=t^{1 / b(\alpha)}$ so that by virtue of the compactness following from (2.14)

$$
\lim _{t \rightarrow \infty} t^{\frac{n(\alpha)}{b(\alpha)}} f_{t \tau}^{t T} f_{\Omega_{R t^{1 / b(\alpha)}}}\left|u(y, s)-E_{\alpha}\left(y_{N}, s\right)\right|^{p} \mathrm{~d} x \mathrm{~d} s=\lim _{k \rightarrow \infty} I_{k}=0 .
$$

Whence (1.22) is proved; (1.23) is proved similarly, on invoking (2.15).

2.2. The outer domain. We define

$$
\Omega^{r}=\Omega \backslash \overline{\Omega_{r}}, \quad \mu_{r}^{k}(t)=\sup _{0<\tau<t} \int_{\Omega^{r}} u_{k}(x, \tau) \mathrm{d} x, \quad \mu_{r}(t)=\mu_{r}^{1}(t) .
$$

where $u_{k}$ is as above and we recall that $u_{1}=u$. We state first the following variant of (2.5).

Lemma 2.3. Under the assumptions of Theorem 1.5 we have for any $t>0$

$$
\left\|u_{k}(t)\right\|_{\infty, \Omega^{R}} \leq \gamma^{*} \delta^{-\omega} \max \left\{\frac{\mu_{(1-\delta) R}^{k}(t)^{\frac{2}{\beta}}}{t^{\frac{N}{\beta}} k^{\lambda(\alpha)}}, \frac{\mu_{(1-\delta) R}^{k}(t)^{\frac{2}{b(\alpha)}}}{t^{\frac{n(\alpha)}{b(\alpha)}}}\right\},
$$

for $0<\delta \leq 1 / 2, \omega=\omega(N)>0$, provided

$$
R \geq C^{*}(N, m, \alpha) \max \left\{\left\|u_{0}\right\|_{1}^{\frac{m-1}{\beta}} t^{\frac{1}{\beta}} k^{-\frac{\beta-b(\alpha)}{\beta}},\left\|u_{0}\right\|_{1}^{\frac{m-1}{b(\alpha)}} t^{\frac{1}{b(\alpha)}}\right\} .
$$

We also need the following estimate.

Lemma 2.4. Under the assumptions of Lemma 2.3 with $C^{*}$ large enough, the following estimate holds

$$
\mu_{R}^{k}(t) \leq \underset{12}{2} \mu_{R / 2}^{k}(0)
$$


The proofs of the two Lemma above are postponed to Section 5 .

We have as a consequence of (2.20) and (2.22) that

$$
\left\|u_{k}(t)\right\|_{\infty, \Omega^{R}} \leq \gamma \max \left\{\frac{\mu_{R / 4}^{k}(0)^{\frac{2}{\beta}}}{t^{\frac{N}{\beta}}}, \frac{\mu_{R / 4}^{k}(0)^{\frac{2}{b(\alpha)}}}{t^{\frac{n(\alpha)}{b(\alpha)}}}\right\}
$$

under assumption (2.21). We select in (2.23) $t=1, k=T^{1 / b(\alpha)}, R=\Gamma$, where $\Gamma$ is the value taken by the right hand side of (2.21) when $t=1$, $k=1$, whence we obtain

$$
T^{\frac{n(\alpha)}{b(\alpha)}}\|u(T)\|_{\infty, \Omega \Gamma T^{1 / b(\alpha)}} \leq \gamma \max \left\{\mu_{\Gamma T^{1 / b(\alpha)} / 4}(0)^{\frac{2}{\beta}}, \mu_{\Gamma T^{1 / b(\alpha)} / 4}(0)^{\frac{2}{b(\alpha)}}\right\} .
$$

Reverting to the name of variable $t$ we have proved that

$$
t^{\frac{n(\alpha)}{b(\alpha)}}\|u(t)\|_{\infty, \Omega \Gamma t^{1 / b(\alpha)}} \rightarrow 0, \quad t \rightarrow \infty .
$$

Next we take into account that $\operatorname{supp} E_{\alpha}(t) \subset B_{R(t)}(0)$, where $R(t)=$ $\gamma C t^{1 / b(\alpha)}$. Since we may assume without loss of generality that $\Gamma \geq \gamma C$, we get

$$
t^{\frac{n(\alpha)}{b(\alpha)}}\left\|u(t)-E_{\alpha}(t)\right\|_{\infty, \Omega^{\Gamma t^{1 / b(\alpha)}}} \rightarrow 0, \quad \text { as } t \rightarrow \infty .
$$

The proof of Theorem 1.5 is concluded.

Proof of Theorem 1.6. The proof in this case proceeds formally along the same lines as above, setting $\alpha=1$. The key difference between the cases of the cone and of the paraboloid is that in the former no essential anisotropy appears in the rescaled equation for $u_{k}$.

Thus the energy inequality in the analogue of Proposition 2.1 is a standard isotropic one, and the limiting function $u_{\infty}$ really depends on all the space variables, solving the standard porous media equation in $\Omega(1)$. This remark motivates the necessity of an assumption controlling the asymptotic behavior of the full matrix of coefficients, as in (1.24).

As a by-product of the stability of the structure of the rescaled equations, we may apply the results of [5] to the sequence $\left\{u_{k}\right\}$, obtaining a uniform modulus of continuity for the functions $u_{k}$. Thus we have uniform convergence to $u_{\infty}=E$ in each bounded set $\Omega_{R} \times(\tau, T), R>0$, $0<\tau<T$, implying by a reasoning similar to the one used above to prove $(1.22)$

$$
\|u(t)-E(t)\|_{\infty,\left\{x_{N}<R t^{1 / \beta}\right\}} \rightarrow 0, \quad t \rightarrow \infty .
$$

The convergence in the outer domain is proved in the same way as in the case $\alpha<1$. 


\section{UNIQUENESS OF THE FUNDAMENTAL SOLUTION.}

Proof of Theorem 1.4. The proof is based on the approach of [16].

Consider for $0<h \leq 1$ the net

$$
u^{h}\left(x_{N}, t\right)=h^{\frac{n(\alpha)}{b(\alpha)}} V\left(h^{\frac{1}{b(\alpha)}} x_{N}, h t\right) .
$$

Notice that by its definition, $u^{h}$ satisfies the same sup bound as in (1.18), and solves (1.12) as well. Also, by mass conservation law (1.20), we have

$$
\int_{0}^{\infty} u^{h}\left(x_{N}, t\right) x_{N}^{\alpha(N-1)} \mathrm{d} x_{N}=\int_{0}^{\infty} V\left(x_{N}, h t\right) x_{N}^{\alpha(N-1)} \mathrm{d} x_{N}=M,
$$

for any $h>0$. On making use of the uniform sup bound recalled above, and of the results in [5], we conclude that $\left\{u^{h}\right\}$ is locally Hölder continuous with a uniform modulus of continuity, so that we may assume, perhaps extracting and relabelling a subsequence, that $u^{h}(\cdot, 1) \rightarrow u_{*}(\cdot)$ as $h \rightarrow 0$ uniformly in any compact subset of $(0, \infty)$.

Let now $t>0$ be fixed, and assume $x_{N} \geq c_{1} t^{1 / b(\alpha)}$, where $c_{1}$ is the constant appearing in (1.19). Then it follows from (1.19) and the definition of $u^{h}$ that $u^{h}\left(x_{N}, t\right)=0$. Thus also the support of $u_{*}(t)$ is contained in $\Omega_{R}$ for $R \geq c_{1} t^{1 / b(\alpha)}$, and we also infer $u^{h}(1) \rightarrow u_{*}$ in $L^{1}((0,+\infty))$ as $h \rightarrow 0$.

Let $v\left(x_{N}, t\right)$ be the solution of problem (1.12)-(1.13) in $(0,+\infty) \times$ $(1,+\infty)$ with initial data $v(1)=u_{*}$; the corresponding modification of Definition 1.3 is straightforward. We remark that the calculations below can then be obtained by approximation since uniqueness of solutions to problems of this kind, with bounded initial data, can be proved along the lines of [19], Chapter 5.

Then by the contraction principle we have for all $t>1$

$$
\begin{aligned}
\int_{0}^{\infty} \mid u^{h}\left(x_{N}, t\right)-v & \left(x_{N}, t\right) \mid x_{N}^{\alpha(N-1)} \mathrm{d} x_{N} \\
& \leq \int_{0}^{\infty}\left|u^{h}\left(x_{N}, 1\right)-v\left(x_{N}, 1\right)\right| x_{N}^{\alpha(N-1)} \mathrm{d} x_{N} \rightarrow 0,
\end{aligned}
$$

as $h \rightarrow 0$. Denote for each $h>0, t>0$,

$$
\omega^{h}(t)=\int_{0}^{\infty}\left|u^{h}\left(x_{N}, t\right)-E_{\alpha}\left(x_{N}, t\right)\right| x_{N}^{\alpha(N-1)} \mathrm{d} x_{N} .
$$


Then the contraction principle yields that $\omega^{h}$ is nonincreasing in time. Moreover by appealing to the selfsimilarity of $E_{\alpha}$ and to the definition of $u^{h}$ we get by a change of integration variable

$$
\omega^{h}(t)=\int_{0}^{\infty}\left|u^{1}\left(y_{N}, h t\right)-E_{\alpha}\left(y_{N}, h t\right)\right| y_{N}^{\alpha(N-1)} \mathrm{d} y_{N}=\omega^{1}(h t),
$$

so that $\omega^{h}(t)$ is nonincreasing both in time and in $h$. Clearly mass conservation implies that $\omega^{h}(t)$ is bounded by $2 M$. Then the following limits exist by monotonicity and are finite

$$
\lim _{h \rightarrow 0} \omega^{h}(1)=\lim _{h \rightarrow 0} \omega^{1}(h)=\lim _{h \rightarrow 0} \omega^{1}(2 h)=\lim _{h \rightarrow 0} \omega^{h}(2)=: \omega^{0} .
$$

Therefore by (3.1)

$$
\omega^{0}=\lim _{h \rightarrow 0} \omega^{h}(1)=\lim _{h \rightarrow 0} \omega^{h}(2)=\int_{0}^{\infty}\left|v\left(x_{N}, 2\right)-E_{\alpha}\left(x_{N}, 2\right)\right| x_{N}^{\alpha(N-1)} \mathrm{d} x_{N} .
$$

We claim that $\omega^{0}=0$. We proceed by contradiction and assume that $\omega^{0}>0$. Define $\bar{u}\left(x_{N}, t\right)$ and $\underline{u}\left(x_{N}, t\right)$ as the solutions of problem (1.12)(1.13) for $t>1$ with initial data

$$
\bar{u}(\cdot, 1)=\max \left\{v(\cdot, 1), E_{\alpha}(\cdot, 1)\right\}, \quad \underline{u}(\cdot, 1)=\min \left\{v(\cdot, 1), E_{\alpha}(\cdot, 1)\right\},
$$

Then by comparison we have

$$
\bar{u} \geq \max \left\{v, E_{\alpha}\right\} \geq \min \left\{v, E_{\alpha}\right\} \geq \underline{u}, \quad \text { in }(0, \infty) \times(1, \infty) .
$$

Since we are assuming that $v(2) \neq E_{\alpha}(2)$, and for $t \geq 1$

$$
\int_{0}^{\infty} E_{\alpha}\left(x_{N}, t\right) x_{N}^{\alpha(N-1)} \mathrm{d} x_{N}=\int_{0}^{\infty} v\left(x_{N}, t\right) x_{N}^{\alpha(N-1)} \mathrm{d} x_{N}=M,
$$

it follows that

$$
\begin{aligned}
& \int_{0}^{\infty}\left(\bar{u}\left(x_{N}, 2\right)-\underline{u}\left(x_{N}, 2\right)\right) x_{N}^{\alpha(N-1)} \mathrm{d} x_{N}> \\
& \int_{0}^{\infty}\left[\max \left\{v, E_{\alpha}\right\}\left(x_{N}, 2\right)-\min \left\{v, E_{\alpha}\right\}\left(x_{N}, 2\right)\right] x_{N}^{\alpha(N-1)} \mathrm{d} x_{N} \\
& \quad=\int_{0}^{\infty}\left|v\left(x_{N}, 2\right)-E_{\alpha}\left(x_{N}, 2\right)\right| x_{N}^{\alpha(N-1)} \mathrm{d} x_{N}=\omega^{0} .
\end{aligned}
$$


On the other hand by the contraction principle

$$
\begin{aligned}
\int_{0}^{\infty} & \left(\bar{u}\left(x_{N}, 2\right)-\underline{u}\left(x_{N}, 2\right)\right) x_{N}^{\alpha(N-1)} \mathrm{d} x_{N} \\
& \leq \int_{0}^{\infty}\left(\bar{u}\left(x_{N}, 1\right)-\underline{u}\left(x_{N}, 1\right)\right) x_{N}^{\alpha(N-1)} \mathrm{d} x_{N} \\
& =\int_{0}^{\infty}\left|v\left(x_{N}, 1\right)-E_{\alpha}\left(x_{N}, 1\right)\right| x_{N}^{\alpha(N-1)} \mathrm{d} x_{N}=\omega^{0},
\end{aligned}
$$

that is an inconsistency. Therefore $\omega^{0}=0$. Since $\omega^{1}(t)$ is nonincreasing in time, we conclude that $\omega^{1}(t)=0$ for all $t>0$.

Corollary 3.1. For $u_{\infty}$ defined as in Section 2, and $E_{\alpha}$ as in Section 1, we have $u_{\infty}=E_{\alpha}$.

Proof. We only need to check that $u_{\infty}$ satisfies the assumptions of Theorem 1.4.

Requirements (1.15) and (1.16) follow by the approximation $u_{k} \rightarrow$ $u_{\infty}$ and from Proposition 2.1 and from (2.9). Equation (1.17) amounts to $(2.19)$.

The bound in (1.18) has been proved in (2.16). As to the support estimate (1.20), we remark that for

$$
R \geq C^{*}\left\|u_{0}\right\|_{1}^{\frac{m-1}{b(\alpha)}} t^{\frac{1}{b(\alpha)}}
$$

where $C^{*}$ is the constant appearing in (2.21), we have by Fatou's lemma

$$
\begin{aligned}
\int_{\Omega^{R}} u_{\infty}\left(y_{N}, t\right) \mathrm{d} y & \leq \liminf _{k \rightarrow \infty} \int_{\Omega^{R}} u_{k}\left(y_{N}, t\right) \mathrm{d} y \\
& \leq 2 \liminf _{k \rightarrow \infty} \int_{\Omega^{R}} u_{k}\left(y_{N}, 0\right) \mathrm{d} y=0 .
\end{aligned}
$$

Indeed we may apply Lemma 2.4 since for large $k$ our assumption (3.2) implies (2.21).

Then conservation of mass as in (1.20) follows again from approximation and from Lemma 2.4. 


\section{Proof of Lemma 2.2.}

4.1. Proof of (2.14). We write, as a consequence of the weak formulation of problem (2.1)-(2.3),

$$
\iint_{S_{T, R, \tau}}\left\{-\left(u_{k}(x, t+h)-u_{k}(x, t)\right) \zeta_{t}+\mathcal{A}_{j}(x, t, h) \zeta_{x_{j}}\right\} \mathrm{d} x \mathrm{~d} t=0,
$$

where we set $R>0, T>\tau>2 h>0$,

$$
\mathcal{A}_{j}(x, t, h)=A_{i j}^{k}(x, t+h)\left(u_{k}^{m}\right)_{x_{i}}(x, t+h)-A_{i j}^{k}(x, t)\left(u_{k}^{m}\right)_{x_{i}}(x, t),
$$

and select for $h>0$

$$
\zeta(x, t)=-\zeta_{1}\left(x_{N}\right) \zeta_{2}(t) \int_{t}^{t+h} u_{k}^{m}(x, s) \mathrm{d} s .
$$

Here $\zeta_{1} \in C_{0}^{1}([0, R)), \zeta_{1}\left(x_{N}\right)=1$ for $0 \leq x_{N} \leq R / 2$, and $\zeta_{2} \in$ $C_{0}^{1}((\tau, T))$ are standard cut-off functions. On using the elementary inequality valid for $a, b \geq 0,\left(a^{m}-b^{m}\right)(a-b) \geq|a-b|^{m+1}$ we find after routine calculations

$$
\begin{aligned}
& \iint_{S_{T, R / 2, \tau}}\left|u_{k}(x, t+h)-u_{k}(x, t)\right|^{m+1} \zeta_{2}(t) \mathrm{d} x \mathrm{~d} t \leq \gamma_{1} h \\
& +\iint_{S_{T, R, \tau}}\left\{\mathcal{A}_{N}(x, t, h) \zeta_{1 x_{N}}\left(x_{N}\right) \zeta_{2}(t) \int_{t}^{t+h} u_{k}^{m}(x, s) \mathrm{d} s\right\} \mathrm{d} x \mathrm{~d} t \\
& +\iint_{S_{T, R, \tau}}\left\{\mathcal{A}_{j}(x, t, h) \zeta_{1}\left(x_{N}\right) \zeta_{2}(t) \int_{t}^{t+h}\left(u_{k}^{m}\right)_{x_{j}}(x, s) \mathrm{d} s\right\} \mathrm{d} x \mathrm{~d} t .
\end{aligned}
$$

Here $\gamma_{1}$ depends on $R, T, \tau,\left\|\zeta_{2 t}\right\|_{\infty}$ and on the bounds in (2.5), but not on $k$.

Next we bound

$$
\begin{array}{r}
\left|\mathcal{A}_{N}(x, t, h) \zeta_{1 x_{N}}\left(x_{N}\right)\right| \leq \gamma\left\{\left|\left(u_{k}^{m}\right)_{x_{N}}(x, t)\right|+\left|\left(u_{k}^{m}\right)_{x_{N}}(x, t+h)\right|\right. \\
\left.+k^{1-\alpha} \sum_{j=1}^{N-1}\left[\left|\left(u_{k}^{m}\right)_{x_{j}}(x, t)\right|+\left|\left(u_{k}^{m}\right)_{x_{j}}(x, t+h)\right|\right]\right\} .
\end{array}
$$

It follows, again taking into account (2.5) and (2.6), that the first integral on the right hand side of (4.2) can be bounded above by $\gamma_{1} h$, perhaps by means of an inessential redefinition of $\gamma_{1}$. 
Finally we turn to estimating the last integral in (4.2); we remark that

$$
\left|\mathcal{A}_{j}(x, t, h) \int_{t}^{t+h}\left(u_{k}^{m}\right)_{x_{j}}(x, s) \mathrm{d} s\right| \leq \gamma G_{0}+\gamma k^{1-\alpha} G_{1}+\gamma k^{2(1-\alpha)} G_{2},
$$

where the quantities $G_{i}$ are as follows, and $\gamma$ is independent of $k$. We have first

$$
G_{0}=\left[\left|\left(u_{k}^{m}\right)_{x_{N}}(x, t)\right|+\left|\left(u_{k}^{m}\right)_{x_{N}}(x, t+h)\right|\right] \times \int_{t}^{t+h}\left|\left(u_{k}^{m}\right)_{x_{N}}(x, s)\right| \mathrm{d} s .
$$

Then we define

$$
\begin{aligned}
G_{1}= & {\left[\left|\left(u_{k}^{m}\right)_{x_{N}}(x, t)\right|+\left|\left(u_{k}^{m}\right)_{x_{N}}(x, t+h)\right|\right] \times \sum_{j=1}^{N-1} \int_{t}^{t+h}\left|\left(u_{k}^{m}\right)_{x_{j}}(x, s)\right| \mathrm{d} s } \\
& +\left[\sum_{j=1}^{N-1}\left[\left|\left(u_{k}^{m}\right)_{x_{j}}(x, t)\right|+\left|\left(u_{k}^{m}\right)_{x_{j}}(x, t+h)\right|\right]\right] \times \int_{t}^{t+h}\left|\left(u_{k}^{m}\right)_{x_{N}}(x, s)\right| \mathrm{d} s,
\end{aligned}
$$

and finally

$$
G_{2}=\sum_{i, j=1}^{N-1}\left[\left[\left|\left(u_{k}^{m}\right)_{x_{i}}(x, t)\right|+\left|\left(u_{k}^{m}\right)_{x_{i}}(x, t+h)\right|\right] \times \int_{t}^{t+h}\left|\left(u_{k}^{m}\right)_{x_{j}}(x, s)\right| \mathrm{d} s\right] .
$$

It follows from a repeated application of Hölder's inequality, and from (2.6), that the last integral in (4.2) is bounded by $\gamma_{1} \sqrt{h}$.

Therefore we have proved (2.14), after a formal redefiniton of $T, \tau$, $R$, and noticing that the case $2 h \geq \tau$ is trivial since the constant in (2.14) is allowed to depend on $\tau$.

4.2. Proof of (2.15). Assume here that $a_{i j}=a_{i j}(x)$ and provisionally that $\partial u_{k} / \partial t \in L_{\mathrm{loc}}^{1}\left(0, T ; L^{1}\left(\Omega_{R}\right)\right)$.

Define

$$
H_{\varepsilon}(s)=\max \{-1, \min (s / \varepsilon, 1)\}, \quad s \in \boldsymbol{R},
$$

so that $H_{\varepsilon}$ is a regular approximation of the function sign. Let $\zeta_{1}$ be as above, while $\zeta_{2}(t)$ vanishes for $t \leq t_{1}-\delta$, and satisfies $\zeta_{2}\left(t_{1}\right)=1$. Here $T \geq t_{1} \geq \tau$ is given, and $t_{1}>\delta>0$ is to be chosen. Define for all relevant functions $f$ and for $0<h<1$

$$
\Delta_{h} f(x, t)=f(x, t+h)-f(x, t) .
$$


We easily obtain for $\mathcal{A}_{j}$ as above

$$
\begin{array}{r}
\int_{0}^{t_{1}} \int_{\Omega_{R}}\left\{\left(\Delta_{h} u_{k}\right)_{t} H_{\varepsilon}\left(\Delta_{h} u_{k}^{m}\right) \zeta_{1} \zeta_{2}+\mathcal{A}_{j}(x, t, h)\left(\Delta_{h} u_{k}^{m}\right)_{x_{j}} H_{\varepsilon}^{\prime}\left(\Delta_{h} u_{k}^{m}\right) \zeta_{1} \zeta_{2}\right. \\
\left.+\mathcal{A}_{N} \zeta_{1 x_{N}} \zeta_{2} H_{\varepsilon}\left(\Delta_{h} u_{k}^{m}\right)\right\} \mathrm{d} x \mathrm{~d} t=0 .
\end{array}
$$

Notice that under our current assumptions

$$
\mathcal{A}_{j}(x, t, h)\left(\Delta_{h} u_{k}^{m}\right)_{x_{j}}=A_{i j}^{k}(x)\left(\Delta_{h} u_{k}^{m}\right)_{x_{i}}\left(\Delta_{h} u_{k}^{m}\right)_{x_{j}} \geq 0 .
$$

Then we drop the corresponding nonnegative term on the left hand side of (4.4) and let $\varepsilon \rightarrow 0$, so that

$$
H_{\varepsilon}\left(\Delta_{h} u_{k}^{m}\right) \rightarrow \operatorname{sign}\left(\Delta_{h} u_{k}^{m}\right)=\operatorname{sign}\left(\Delta_{h} u_{k}\right), \quad \text { for a.e. }(x, t) .
$$

Then, on integrating by parts in time, we get

$$
\begin{aligned}
& \int_{\Omega_{R}}\left|\left(\Delta_{h} u_{k}\right)\left(x, t_{1}\right)\right| \zeta_{1}\left(x_{N}\right) \mathrm{d} x \leq \gamma \int_{t_{1}-\delta} \int_{\Omega_{R}}^{t_{1}}\left\{\left|\left(\Delta_{h} u_{k}^{m}\right)_{x_{N}}\right|\right. \\
& \left.\quad+k^{1-\alpha} \sum_{i=1}^{N-1}\left|\left(\Delta_{h} u_{k}^{m}\right)_{x_{i}}\right|\right\} \mathrm{d} x \mathrm{~d} t+\gamma \delta^{-1} \int_{t_{1}-\delta}^{t_{1}} \int_{\Omega_{R}}\left|\Delta_{h} u_{k}\right| \mathrm{d} x \mathrm{~d} t .
\end{aligned}
$$

The first integral on the right hand side of (4.5) can be bounded by means of (2.6) and of Hölder's inequality, obtaining the majorant $\gamma \sqrt{\delta}$.

On applying again Hölder's inequality, the last term on the right hand side of (4.5) can be bounded by

$$
\gamma \delta^{-1} \delta^{\frac{m}{m+1}}\left[\int_{t_{1}-\delta}^{t_{1}} \int_{\Omega_{R}}\left|\Delta_{h} u_{k}\right|^{m+1} \mathrm{~d} x \mathrm{~d} t\right]^{\frac{1}{m+1}} \leq \gamma \delta^{-\frac{1}{m+1}} h^{\frac{1}{2(m+1)}},
$$

where we have made use of (2.14). Collecting the estimates above, and selecting $\delta=h^{1 /(m+3)}$ we obtain (substituting $t_{1}$ with the more usual variable $t$ )

$$
\int_{\Omega_{R / 2}}\left|u_{k}(x, t+h)-u_{k}(x, t)\right| \mathrm{d} x \leq \gamma h^{\frac{1}{2(m+3)}}, \quad t \in[\tau, T],
$$

where $\gamma$ depends on $R$ and on $\tau, T$, but not on $k$. We also have to assume for example $h<\tau^{2(m+3)} / 3$ for the selection of $\delta$ as above to be possible, but the case where the converse holds true is in fact trivial, as remarked above. 
The extra assumption on the integrability of the time derivative of $u_{k}$ may be removed by approximation. In fact $u$ and therefore $u_{k}$ are limits of a sequence $\left\{v_{k n}\right\}_{n \geq 1}$ of compactly supported solutions: we can prove (4.6) for each $v_{k n}$ and then take the limit $n \rightarrow \infty$. Indeed, under our current stipulation $a_{i j}=a_{i j}(x)$, each $\partial v_{k n} / \partial t$ can be proved to be integrable as required by means of the same approach as in [19].

\section{Proof of the Localized estimates.}

Proof of Lemma 2.3. The inequality

$$
\|u(T)\|_{\infty, \Omega^{\rho}} \leq \gamma^{*} \delta^{-\omega} \max \left\{\frac{\mu_{(1-\delta) \rho}(T)^{\frac{2}{\beta}}}{T^{\frac{N}{\beta}}}, \frac{\mu_{(1-\delta) \rho}(T)^{\frac{2}{b(\alpha)}}}{T^{\frac{n(\alpha)}{b(\alpha)}}}\right\},
$$

follows as in Lemma 3.1 of [3], see also Remark 3.1 there, provided

$$
\frac{T}{\rho^{2}} \sup _{T / 2<\tau<T}\|u(\tau)\|_{\infty}^{m-1} \leq 1 .
$$

In fact in [3] the localization is performed in $\Omega_{r}$ rather than in $\Omega^{r}$, but this technical difference is irrelevant under our assumptions of global integrability of the solution in space. Indeed, we have available the global estimate (1.9), which allows us to infer condition (5.2) from

$$
\rho \geq \gamma \max \left\{\left\|u_{0}\right\|_{1}^{\frac{m-1}{\beta}} T^{\frac{1}{\beta}},\left\|u_{0}\right\|_{1}^{\frac{m-1}{b(\alpha)}} T^{\frac{1}{b(\alpha)}}\right\} .
$$

We use (5.1) with $\rho, T$ replaced with $R k, k^{b(\alpha)} t$. One sees that the necessary condition (5.3) follows now from assumption (2.21). Finally, the statement follows from this version of (5.1) and the definition of $u_{k}$.

Proof of Lemma 2.4. Let $R_{n}=R\left(1-2^{-n}\right), n \geq 1, \bar{R}_{n}=\left(R_{n}+R_{n+1}\right) / 2$ and let $\zeta_{n}\left(x_{N}\right)$ be a smooth cutoff function such that $\zeta_{n}\left(x_{N}\right)=1$ for $x_{N} \geq R_{n+1}, \zeta_{n}\left(x_{N}\right)=0$ for of $x_{N} \leq \bar{R}_{n},\left|\zeta_{n x_{N}}\right| \leq \gamma 2^{n} R^{-1}$. On multiplying both sides of equation (1.1) by $\zeta_{n}^{2}$ and integrating by parts over $\Omega$, we get

$$
\begin{aligned}
\int_{\Omega} \zeta_{n}\left(x_{N}\right)^{2} u(x, t) \mathrm{d} x= & \int_{\Omega} \zeta_{n}\left(x_{N}\right)^{2} u(x, 0) \mathrm{d} x \\
& \quad-2 m \int_{0}^{t} \int_{\Omega} \zeta_{n} u^{m-1} a_{i N} u_{x_{i}} \zeta_{n x_{N}} \mathrm{~d} x \mathrm{~d} \tau .
\end{aligned}
$$


Applying Hölder's inequality we bound the double integral in (5.4) from above by

$$
\begin{gathered}
\gamma\left(\int_{0}^{t} \int_{\Omega} \zeta_{n}^{2} \tau^{\mu} u^{-\theta+m-1}|\nabla u|^{2} \mathrm{~d} x \mathrm{~d} \tau\right)^{\frac{1}{2}} \\
\times\left(\int_{0}^{t} \int_{\Omega} \zeta_{n x_{N}}^{2} \tau^{-\mu} u^{\theta+m-1} \mathrm{~d} x \mathrm{~d} \tau\right)^{\frac{1}{2}}=: J_{1}^{\frac{1}{2}} J_{2}^{\frac{1}{2}} .
\end{gathered}
$$

The parameters $\mu>0$ and $\theta<1$ will be chosen presently. Next in order to bound $J_{1}$ in (5.5) we multiply both sides of (1.1) by $\zeta_{n}^{2} \tau^{\mu} u^{1-\theta}$ and integrate by parts. Then after standard calculations we get

$$
J_{1} \leq \gamma \int_{0}^{t} \int_{\Omega^{\bar{R}_{n}}} \tau^{\mu-1} u^{2-\theta} \mathrm{d} x \mathrm{~d} \tau+\gamma \int_{0}^{t} \int_{\Omega} \tau^{\mu} \zeta_{n x_{N}}^{2} u^{-\theta+m+1} \mathrm{~d} x \mathrm{~d} \tau .
$$

We now appeal to the $L^{1}-L^{\infty}$ estimate

$$
\|u(t)\|_{\infty, \Omega^{\bar{R}_{n}}} \leq \gamma b^{n} \max \left\{\frac{\mu_{R_{n}}(t)^{\frac{2}{\beta}}}{t^{\frac{N}{\beta}}}, \frac{\mu_{R_{n}}(t)^{\frac{2}{\beta(\alpha)}}}{t^{\frac{N(\alpha)}{\beta(\alpha)}}}\right\}=: \gamma b^{n} B_{n}(t),
$$

where $b=b\left(c_{0}, N, m, \alpha\right)>1$, which is essentially (5.1), and is valid under the assumption (5.2) where we replace $\rho, T$ with $R, t$ respectively. Notice that this assumption is therefore nothing else than (2.21) with $k=1$.

We choose $N(m-1) / \beta<\mu<1$ and $\theta=2-m$, and obtain from $(5.6)-(5.7)$

$$
\begin{aligned}
J_{1} & \leq \gamma \mu_{R_{n}}(t) \int_{0}^{t}\left[\|u(\tau)\|_{\infty, \Omega^{R_{n}}}^{m-1} \tau^{\mu-1}+\frac{2^{2 n}}{R^{2}}\|u(\tau)\|_{\infty, \Omega^{R_{n}}}^{2(m-1)} \tau^{\mu}\right] \mathrm{d} \tau \\
& \leq \gamma b^{n} \mu_{R_{n}}(t) \int_{0}^{t}\left[B_{n}(\tau)^{m-1} \tau^{\mu-1}+R^{-2} B_{n}(\tau)^{2(m-1)} \tau^{\mu}\right] \mathrm{d} \tau \\
& \leq \gamma b^{n} \mu_{R_{n}}(t)\left[t^{\mu} B_{n}(t)^{m-1}+R^{-2} t^{1+\mu} B_{n}(t)^{2(m-1)}\right] \\
& =\gamma b^{n} \mu_{R_{n}}(t) t^{\mu} B_{n}(t)^{m-1}\left[1+R^{-2} t B_{n}(t)^{m-1}\right] \leq \gamma b^{n} \mu_{R_{n}}(t) t^{\mu} B_{n}(t)^{m-1},
\end{aligned}
$$

where the last inequality follows from the trivial bound $\mu_{R_{n}}(t) \leq\left\|u_{0}\right\|_{1}$, and from our assumption (2.21) with $k=1$. An even simpler argument 
shows that

$$
J_{2} \leq b^{n} R^{-2} \mu_{R_{n}}(t) t^{1-\mu} .
$$

Thus, collecting the estimates just found for $J_{1}$ and $J_{2}$ we get

$$
\begin{aligned}
\mu_{R_{n+1}}(t) & \leq \int_{\Omega^{R / 2}} u_{0} \mathrm{~d} x \\
& +b^{n} R^{-1} \mu_{R_{n}}(t)^{1+\frac{m-1}{\beta}} \max \left\{t^{\frac{1}{\beta}}, t^{\frac{1}{b(\alpha)}} \mu_{R_{n}}(t)^{\frac{m-1}{b(\alpha)}-\frac{m-1}{\beta}}\right\} .
\end{aligned}
$$

We reason now by contradiction, and assume the majorization

$$
\mu_{R}(t)=\lim _{n \rightarrow \infty} \mu_{R_{n}}(t)=\inf _{n \geq 1} \mu_{R_{n}}(t)>2 \int_{\Omega^{R / 2}} u_{0} \mathrm{~d} x .
$$

Then from (5.8) we have

$$
\mu_{R_{n+1}}(t) \leq 2 b^{n} R^{-1} \mu_{R_{n}}(t)^{1+\frac{m-1}{\beta}} \max \left\{t^{\frac{1}{\beta}}, t^{\frac{1}{b(\alpha)}}\left\|u_{0}\right\|_{1}^{\frac{m-1}{b(\alpha)}-\frac{m-1}{\beta}}\right\} .
$$

Therefore according to the well known iterative Lemma 5.6 Chap. II of [14] we have that $\mu_{R_{n}}(t) \rightarrow 0$ as $n \rightarrow \infty$ provided

$$
R^{-1} \mu_{R_{1}}(t)^{\frac{m-1}{\beta}} \max \left\{t^{\frac{1}{\beta}}, t^{\frac{1}{b(\alpha)}}\left\|u_{0}\right\|_{1}^{\frac{m-1}{b(\alpha)}-\frac{m-1}{\beta}}\right\} \leq \gamma_{0}
$$

which is in turn implied by, when we recall that $\mu_{R_{1}}(t) \leq\left\|u_{0}\right\|_{1}$,

$$
R^{-1} \max \left\{t^{\frac{1}{\beta}}\left\|u_{0}\right\|_{1}^{\frac{m-1}{\beta}}, t^{\frac{1}{b(\alpha)}}\left\|u_{0}\right\|_{1}^{\frac{m-1}{b(\alpha)}}\right\} \leq \gamma_{0} .
$$

This inequality is exactly (2.21) with $k=1$, perhaps after an inessential redefinition of $C^{*}$ there. Since we have proved that assumption (5.9) leads us to an inconsistency, we conclude that

$$
\mu_{R}(t)=\int_{\Omega^{R}} u(x, t) \mathrm{d} x \leq 2 \int_{\Omega^{R / 2}} u_{0}(x) \mathrm{d} x .
$$

Finally (2.22) follows by replacing $R$ with $k R$ and $t$ with $k^{b(\alpha)} t$ in (5.10), which is possible provided

$$
(k R)^{-1} \max \left\{\left(k^{b(\alpha)} t\right)^{\frac{1}{\beta}}\left\|u_{0}\right\|_{1}^{\frac{m-1}{\beta}},\left(k^{b(\alpha)} t\right)^{\frac{1}{b(\alpha)}}\left\|u_{0}\right\|_{1}^{\frac{m-1}{b(\alpha)}}\right\} \leq \gamma_{0} .
$$

However this inequality follows from (2.21). 


\section{REFERENCES}

[1] N. D. Alikakos and R. Rostamian. On the uniformization of the solutions of the porous medium equation in $\mathbf{R}^{N}$. Israel J. Math., 47(4):270-290, 1984.

[2] D. Andreucci and A. F. Tedeev. Optimal bounds and blow up phenomena for parabolic problems in narrowing domains. Proceedings Royal Soc. Edinburgh, 128A:1163-1180, 1998. RSE Scotland Foundation, Edinburgh (UK).

[3] D. Andreucci and A. F. Tedeev. A Fujita type result for a degenerate Neumann problem in domains with non compact boundary. J. Math. Analysis and Appl., 231:543-567, 1999. Elsevier.

[4] D. Andreucci and A. F. Tedeev. Sharp estimates and finite speed of propagation for a Neumann problem in domains narrowing at infinity. Advances Diff. Eqs., 5:833-860, 2000. Khayyam Publ., Athens Ohio (U.S.A.).

[5] E. DiBenedetto. Continuity of weak solutions to a general porous medium equation. Indiana Univ. Math. J., 32(1):83-118, 1983.

[6] S. D. Eidelman, S. Kamin, and A. F. Tedeev. On stabilization of solutions of the Cauchy problem for linear degenerate parabolic equations. Adv. Differential Equations, 14(7-8):621-641, 2009.

[7] G. Grillo and M. Muratori. Sharp short and long time $L^{\infty}$ bounds for solutions to porous media equations with homogeneous Neumann boundary conditions. J. Differential Equations, 254(5):2261-2288, 2013.

[8] A. K. Gushchin. The rate of stabilization of a solution of a parabolic equation in an unbounded region. Diff. Uravn., 6:741-761, 1970. Engl. Transl. Diff. Eqns. $6,1970,567-581$.

[9] A. K. Gushchin. On estimates of solutions of boundary value problems for for a second order parabolic equation. Trudy Mat. Inst. Steklov, 126:5-45, 1973. Engl. Transl. Proc. Steklov Inst. Math. 126, 1973.

[10] A. K. Gushchin. On the uniform stabilization of solutions of the second mixed problem for a parabolic equation. Mat. Sbornik, 119(161), 1982. Engl. Transl. Math. USSR Sbornik 47, 1984, 439-498.

[11] S. Kamin. On stabilisation of solutions of the Cauchy problem for parabolic equations. Proc. Roy. Soc. Edinburgh Sect. A, 76(1):43-53, 1976/77.

[12] S. Kamin and L. A. Peletier. Large time behaviour of solutions of the porous media equation with absorption. Israel J. Math., 55(2):129-146, 1986.

[13] S. Kamin and J. L. Vázquez. Fundamental solutions and asymptotic behaviour for the p-Laplacian equation. Rev. Mat. Iberoamericana, 4(2):339-354, 1988.

[14] O. A. Ladyzhenskaja, V. A. Solonnikov, and N. N. Ural'ceva. Linear and Quasilinear Equations of Parabolic Type, volume 23 of Translations of Mathematical Monographs. American Mathematical Society, Providence, RI, 1968.

[15] M. Pierre. Uniqueness of the solutions of $u_{t}-\Delta \varphi(u)=0$ with initial datum a measure. Nonlinear Anal., 6(2):175-187, 1982.

[16] Y. Qi and M. Wang. Singular solutions of doubly singular parabolic equations with absorption. Electron. J. Differential Equations, pages No. 67, 22 pp. (electronic), 2000.

[17] P. Rosenau and S. Kamin. Nonlinear diffusion in a finite mass medium. Comm. Pure Appl. Math., 35(1):113-127, 1982.

[18] J. L. Vázquez. Asymptotic beahviour for the porous medium equation posed in the whole space. J. Evol. Equ., 3(1):67-118, 2003. Dedicated to Philippe Bénilan. 
[19] J. L. Vázquez. The porous medium equation. Oxford Mathematical Monographs. The Clarendon Press, Oxford University Press, Oxford, 2007. Mathematical theory.

Department of Basic and Advanced Sciences for Engineering, Sapienza University of Rome, via A.Scarpa 16, 00161 Roma Italy

Email address: daniele.andreucci@sbai.uniroma1.it

South Mathematical Institute of VSC RAS, Vladikavkaz, 362027, Vladikavkaz, Markusa, 22 Russia

Email address: a_tedeev@yahoo.com 